\title{
Civilisations
}

Revue internationale d'anthropologie et de sciences

humaines

42-1 | 1993

Amérique latine-Europe

\section{La dette, les ajustements et le commerce de l'Amérique latine face à l'Europe}

\section{Marcelo Ossandon}

\section{OpenEdition \\ Journals}

Édition électronique

URL : http://journals.openedition.org/civilisations/2111

DOI : 10.4000/civilisations. 2111

ISSN : 2032-0442

\section{Éditeur}

Institut de sociologie de l'Université Libre de Bruxelles

\section{Édition imprimée}

Date de publication : 1 novembre 1993

Pagination : 203-220

ISBN : 0009-8140

ISSN : 0009-8140

Référence électronique

Marcelo Ossandon, « La dette, les ajustements et le commerce de l'Amérique latine face à l'Europe », Civilisations [En ligne], 42-1 | 1993, mis en ligne le 30 novembre 1996, consulté le 19 avril 2019. URL http://journals.openedition.org/civilisations/2111; DOI : 10.4000/civilisations.2111

Ce document a été généré automatiquement le 19 avril 2019

(c) Tous droits réservés 


\title{
La dette, les ajustements et le commerce de l'Amérique latine face à l'Europe
}

\author{
Marcelo Ossandon
}

1 La décennie des années 90 présente des changements substantiels pour l'Europe, qui auront sans doute des répercussions importantes dans les relations politiques, économiques et sociales de la Communauté européenne avec l'Amérique latine.

2 Les bouleversements dans l'Europe de l'Est, l'éclatement de l'ancienne Union Soviétique, la guerre civile qui s'enlise en Yougoslavie, la montée des nationalismes, et d'une manière générale, la faillite du socialisme dit réel, font penser que l'Amérique latine risque d'occuper une place assez secondaire dans les priorités de la Communauté.

Par ailleurs, les balbutiements de la politique de défense et de sécurité extérieure de l'Europe des douze, ses va-et-vient dans la construction de l'union économique (songeons au NON danois et au timide OUI français) avec des secousses dans le système monétaire qui ébranlent les monnaies du Royaume-Uni, de l'Espagne et de l'Italie, entraîneront inévitablement des répercussions économiques, politiques et sociales dans les relations de la Communauté avec le reste du monde.

Dans le cadre de ce nouveau rôle international, qui reste toujours à définir, quelles politiques de coopération avec le monde en développement, et notamment avec l'Amérique latine, l'Europe mettra-t-elle en oeuvre?

Cette interrogation nous place d'emblée entre l'illusion et le réalisme concernant les relations Europe-Amérique latine (Van Klaveren A., 1991)1. Du côté de l'illusion, les profonds liens historiques noués avec l'Europe devraient s'exprimer également au niveau économique. Politiquement, d'importants secteurs latino-américains eurent tendance à considérer l'Europe occidentale comme une troisième voie entre les deux superpuissances, un contrepoids aux Etats-Unis. Du côté réalisme, les bouleversements en Europe de l'Est ont amené les dirigeants politiques à déplorer l'oubli de l'Amérique latine par ses partenaires européens. Il semblerait que la tendance interne à la Communauté 
européenne, comme dans les autres pays du Nord, est de dévier une partie de l'aide qui était affectée au Sud vers le développement de l'Est.

6 Sans doute, le problème de la dette des principaux pays latino-américains a introduit des facteurs d'inhibition dans les liens entre les deux régions. Si, pour certains, la crise de la dette semble résolue d'un point de vue bancaire, dans la mesure où les risques de faillite du système financier se sont éloignés par rapport à 1982, le thème reste d'une actualité brûlante dans l'optique latino- américaine.

7 C'est pourquoi le thème doit être analysé dans un cadre plus large. Nous songeons notamment au contexte extérieur des relations économiques du continent avec le capitalisme développé et tout particulièrement avec l'Europe des douze.

La dette (extérieure, sociale et écologique), les diverses difficultés dans le secteur extérieur et l'ensemble des problèmes internes (dont l'environnement), apparaissent étroitement liés. En définitive, chacun d'eux est l'expression d'une crise plus générale qui les inclut.

De plus, la relative similitude avec laquelle s'expriment les signes de la crise dans les différentes économies nationales, malgré les variantes notoires de pays à pays, tant politiques qu'économiques, a motivé des réponses semblables, que l'on a appelées ajustements structurels.

10 Ainsi, notre contribution s'articule autour des aspects suivants: dette, ajustements structurels, commerce en Amérique latine. L'ampleur du sujet, nous amène à lui donner fatalement un caractère schématique, tout en essayant d'ajouter des éléments concernant les relations Europe-Amérique latine.

\section{La dette extérieure}

\section{L'historique}

11 L'influence de l'environnement extérieur sur l'évolution économique de la région a toujours été considérable. Rappelons qu'au XIX siècle déjà le continent enregistrait un endettement élevé. L'Amérique latine était, par comparaison à d'autres régions sousdéveloppées, la plus grande consommatrice de capitaux des pays industrialisés (Corm G, 1992)2. Sans qu'il soit besoin de remonter aux économies primaires d'exportation et d'approfondir les modalités du processus d'industrialisation par substitution des importations conformément aux théories de la CEPAL (Prebisch R, 1959)3), les situations les plus récentes avalisent cette assertion. Si les 25 ans qui ont précédé l'effondrement du système de Bretton Woods ont été un âge d'or pour les économies industrielles de l'Occident et, indirectement, pour le Tiers-Monde ; à partir de 1973, le taux d'expansion du commerce et de l'activité économique mondiale s'est trouvé réduit de moitié. La première crise pétrolière provoque cependant une récession de courte durée dans la mesure où, dans les pays sous-développés, les effets de contraction de la demande sur le marché mondial sont atténués par un fait capital : la capacité, sans précédent pour les pays du Tiers-Monde, de s'endetter sur le marché des capitaux privés à des taux d'intérêts réels relativement bas, conséquence du faible cours du dollar et des hauts niveaux d'inflation mondiale. Ce processus crée les prémisses de l'endettement (Ossandon M, 1991, Bergel S, 1993) ${ }^{4}$.

Ces prémisses sont renforcées par deux faits caractérisant les échanges internationaux : 
1. Les investissements des pays capitalistes développés qui, avant la $2^{\text {ème }}$ guerre, s'adressaient au monde colonial, semi colonial ou périphérique, commencent à se faire essentiellement entre pays développés.

2. Au début des années 70, on constate un accroissement du capital privé dans l'ensemble des flux financiers vers les pays sous-développés. Dans ce cadre, les investissements directs diminuent en faveur des prêts et des crédits.

On en arrive à une crise systémique de surendettement, dans laquelle les différents acteurs agissent « rationnellement » en fonction de la logique du marché :

- les pays s'endettent alors que prévalent des taux d'intérêt réels négatifs;

- les banques placent leurs excédents dans les pays qui les sollicitent;

- les organismes internationaux considèrent les banques commerciales comme une source d'appui insoupçonnée ;

- les spéculateurs profitent des règles du jeu particulièrement favorables qui leur sont offertes (Rimez M, 1991) ${ }^{5}$.

13 C'est pourquoi on met en avant le principe de coresponsabilitét. On connaît bien les facteurs qui, à partir de 1981, provoquent une diminution de l'activité économique mondiale :

1. Une réduction de la demande de produits du Tiers-Monde, notamment de leurs exportations de produits de base et de matières premières.

2. Une chute des prix de ces produits, conséquence de la réduction de la demande et, partant, des variations fâcheuses des termes de l'échange.

3. Une réduction de l'aide internationale et autres apports de capitaux (Singh A, 1986) ${ }^{7}$.

Cette situation coïncide pour l'Amérique latine avec la chute brutale du volume de prêts (Ossandon M, 1985 et 1991) ${ }^{8}$ qui accélère la crise, et fait reculer de 15 ou 20 ans le niveau de vie de nombreux pays. Dès lors, les années 80 seront considérées comme une décennie perdue pour l'Amérique latine.

\section{Les acteurs externes}

14 A partir de 1979, la Réserve fédérale américaine (FED) préconise une politique monétaire restrictive, particulièrement aux Etats-Unis où l'inflation avait atteint des niveaux sans précédent. La forte hausse des taux d'intérêt, conséquence du déficit américain, et de la réévaluation du dollar parvinrent à freiner les tendances inflationnistes, mais au prix d'une récession qui s'est étendue, sur une plus ou moins grande échelle, à tous les pays de l'OCDE.

15 L'instabilité et l'incertitude, implicites dans les nouvelles modalités selon lesquelles les taux d'intérêt et les taux de change acquièrent un rôle croissant comme variables d'ajustement dans les relations commerciales entre pays, ont fait que "l'ordre ancien " de Bretton Woods, en vigueur jusqu'en 1971, ait été progressivement remplacé par un système " d'instabilités contrôlées ".

16 Face au cours élevé du dollar, aux taux d'intérêts en hausse, à la dégradation constante des termes de l'échange des produits latino-américains, aux mesures protectionnistes des pays développés, y compris la CE, nous pouvons donc affirmer qu'un ensemble des facteurs agissant simultanément explique le phénomène croissant de l'endettement latino-américain.

17 La phase d'abondance des ressources financières ayant pris fin brusquement, les débiteurs commencent à éprouver de sérieuses difficultés pour assurer le service de la 
dette. La Banque transnationale réduit les prêts et les créditeurs se préoccupent exclusivement de la capacité des pays endettés à faire face à leurs obligations.

Il revient au binôme FMI-Banque mondiale de faire pression sur ces pays, dans la mesure où les banques privées n'ont pas le pouvoir direct pour y arriver.

On sait que le FMI conditionne l'accès aux nouveaux crédits à la mise en oeuvre, par les pays débiteurs, de plans d'austérité et d'ajustements économiques: dévaluation, compression salariale, contraction des budgets sociaux constituant l'essentiel de ces politiques.

Outre le fait que ces plans menacent de déboucher sur de graves conflits sociaux, ces politiques d'ajustement et de stabilisation ont pris le pas sur les politiques de développement. Ainsi, des efforts plus ou moins réussis pour s'adapter aux contraintes d'une économie mondiale dominée par les grands pays industrialisés ont remplacé les stratégies de développement à long terme.

21 En effet, le premier objectif de l'ajustement, appellation officielle de l'austérité, est par conséquent d'accumuler assez de devises pour permettre le remboursement des prêts. C'est seulement s'il a été capable de remplir l'essentiel des obligations du service de sa dette qu'un pays débiteur est considéré par le FMI libre de poursuivre d'autres objectifs. Le débiteur n'a d'autre choix que de suivre les avis du Fonds puisque, sans son approbation, aucun autre bailleur de fonds ne lui consentira de prêts, ni même de crédits commerciaux à court terme.

Par ailleurs, on assiste aussi à un impressionnant réalignement idéologique des organisations internationales outre que le FMI (BM, CE) dans la nécessité d'un ajustement aux réalités de marchég.

Quant à l'Amérique latine, la question qui se pose est de savoir s'il existe une alternative au modèle libéral ou néo-libéral. L'idée de «moins d'Etat » est défendue du Nord au Sud de l'Amérique latine, conséquence de l'échec de l'Etat depuis l'époque du populisme jusqu'à aujourd'hui, à construire une société moins inégale (Marques-Pereira J., 1992) ${ }^{10}$. On constate la disparition de toute politique de rechange qui n'emprunterait pas les voies tracées par le libéralisme, alors que ces alternatives existaient dans les années 70.

Ce retour en force aux lois du marché devient une sorte de panacée universelle, alors que l'expérience latino-américaine montre amplement qu'il ne suffit pas de laisser au marché rétablissement des prix, pour qu'automatiquement les économies enregistrent un processus continu de croissance et de développement. Rappelons à cet égard que, pour une série de problèmes de société, le marché s'est révélé incapable de trouver des solutions. L'extrême pauvreté, les catastrophes écologiques, l'inégale répartition de la richesse et l'absence de justice sociale constituent autant d'exemples qui attestent de cette impuissance.

Bref, la nécessité de la coopération et de la solidarité en dehors du marché, ainsi que la mise sur pied de nouvelles formes de régulation et d'intervention de l'Etat, demeurent comme objectifs prioritaires à redéfinir, avant même de parler de commerce et d'ouverture des économies latino-américaines.

L'Europe des douze aidera-t-elle l'Amérique latine à surmonter la crise qu'elle traverse, alors que la plupart des pays latino-américains ouvrent unilatéralement leurs marchés intérieurs? 


\section{Le FMI et les politiques d'ajustements structurels}

27 Le FMI, dans le but de permettre un libre fonctionnement du marché dans les pays où il intervient, et dans la ligne des ajustements passifs vis-à-vis des nécessités de l'économie mondiale, impose d'importantes modifications dans les relations entre acteurs économiques.

Toute l'analyse du FMI et de la Banque mondiale tente de modifier l'économie interne d'un pays, car celle-ci serait la responsable des déséquilibres externes, à partir d'une optique éminemment monétariste de la balance des paiements.

Une description sommaire de ces mesures inclut:

- suppression ou réduction des subventions aux prix afin de libérer des ressources pour les utiliser de manière plus adéquate ;

- libéralisation des prix agricoles, pour favoriser les producteurs ;

- élimination des barrières à l'importation pour favoriser l'investissement, non pas vers les secteurs protégés, mais plutôt vers ceux qui assurent les exportations;

- taux d'intérêt réels élevés, afin de stimuler l'épargne locale ;

- dévaluation de la monnaie nationale pour encourager les exportations;

- élimination des obstacles au transfert de capitaux (par ex. les bénéfices des multinationales).

Les programmes sociaux contre la pauvreté sont souvent réalisés en décalage avec ces mesures économiques. Résultat des courses de cette conception : la crise dans les pays historiquement situés dans la périphérie est attribuée, de manière univoque, à l'épuisement des "modèles de développement" propres à ces pays. Ainsi, la crise apparait comme étrangère aux effets de l'intégration de chaque économie et de chaque société dans un système mondial que nous savons hiérarchisé, avec tout ce que cela implique en termes de flux de capitaux, de biens, de différence de productivité, de dépendance technologique, militaire et culturelle, d'alliances entre les élites du Sud et du Nord (André Udry Ch., 1991) ${ }^{11}$.

Pour le FMI, chaque pays est considéré comme une unité indépendante, régie par des normes macro-économiques fondamentalement analogues. D'où les recettes similaires pour tous les pays.

31 Depuis plus de 20 ans, l'Amérique latine associe l'épuisement des modèles de croissance, le modèle de substitution des importations et les limitations du contexte extérieur, en particulier le problème de la dette extérieure.

Il est vrai que l'Amérique latine a appliqué durant des années le dogme de la substitution des importations, avec une excessive intervention de l'Etat qui a conduit à une certaine paralysie économique. Sans aucun doute, il était nécessaire de corriger cette approche. Mais, était-il inévitable de le faire, comme au Venezuela, en Argentine, en Bolivie ou au Pérou, au moyen d'une thérapie brutale? A quels risques soumet-on ainsi les fragiles démocraties latino-américaines? (Ramonet I., 1992) ${ }^{12}$.

En ce qui concerne l'épuisement du modèle, les répartitions inégales du revenu ont eu des effets négatifs sur le marché intérieur. Le coût social des ajustements a été répercuté de manière disproportionnée sur les travailleurs et les couches à revenu moyen, qui ont assumé le gros des sacrifices associés au syndrome de la stagnation avec inflation.

Le manque de dynamisme économique, les modifications dans la situation de l'emploi, accompagnées de détériorations du salaire réel, et les restrictions des dépenses publiques 
ont contribué conjointement à l'augmentation de l'extrême pauvreté. A de rares exceptions près, tous les secteurs publics sont entrés en crise dans les années 80 . A la demande croissante dans les domaines de l'éducation et de la santé, les restrictions financières du secteur de l'Etat ont provoqué d'importantes réductions des dépenses par habitant. Par contre, les dépenses courantes ont plutôt tendance à augmenter, en partie comme conséquence du paiement des intérêts de la dette publique, intérieure et extérieure. La charge financière qui découle de cette dernière, exprimée en monnaie nationale, s'est vue amplifiée par des dévaluations successives et des taux d'intérêt élevés. A cette vision négative d'autres auteurs opposent les atouts principaux de la région (Van Klaveren, 1991) ${ }^{13}$.

L'abondance de ressources naturelles, comprenant un secteur agricole doté de puissants avantages comparatifs, un secteur industriel non négligeable avec des cadres techniques et administrables de qualité. Les avantages relatifs, joints aux facilités prévues pour la conversion de la dette extérieure en actions d'entreprises locales, ont permis un important retour des investissements étrangers tant directs qu'indirects, dans lesquels des investisseurs européens ont participé de manière notable.

\section{Le marché secondaire de la dette}

\section{Le rôle des banques européennes}

La dette extérieure contractée par les pays latino-américains auprès des banques européennes, représentant $35 \%$ de l'endettement total de la région auprès des banques privées internationales, a constitué un obstacle à des relations économiques plus étroites entre les deux régions (Grandi J., 1991) ${ }^{14}$.

Lorsque la crise de la dette éclata en 1992, les banques d'Europe continentale ont dû suivre la stratégie de "gestion de la crise » définie par les grandes banques américaines. Cependant, cette gestion se réalise dans un contexte de concurrence exacerbée. Or, en 1989, la dette des pays latino-américains avec les banques commerciales de six pays européens (Allemagne, France, Italie, Pays-Bas, Royaume-Uni et Suisse) s'élevait à 81,5 milliards de dollars, contre 58,9 milliards vis-à-vis des banques des Etats-Unis (Suarez, S., 1991) $)^{15}$.

$\mathrm{Si}$, dans un premier temps, les grandes banques américaines, relativement plus engagées, ont imposé leur stratégie de restructuration aux banques européennes, celles-ci ont mené simultanément une politique systématique d'approvisionnement et de restructuration de leurs portefeuilles (désengagement, mais également diversification ou concentration des créances), à travers le "marché secondaire des dettes " qu'elles ont mis en place dès 1982-1983. Si les grandes banques américaines réaffirment qu'un Etat souverain «ne disparaît pas " et que les problèmes transitoires de liquidités se résolvent par un ajustement rapide des économies débitrices, les banques d'Europe, au contraire, notamment la Deutsche Bank, n'excluaient pas cette hypothèse. Les banques françaises et allemandes affirment alors la nécessité d'une gestion à long terme, l'insuffisance des rééchelonnements pour résoudre la crise, et, surtout, expriment leur opposition au principe de "new money " (prêt involontaire servant au paiement des intérêts dus) qui sont davantage utiles aux banques américaines qu'aux pays bénéficiaires. Le niveau de provisions et le traitement de celles-ci, qui varie de pays à pays expliquerait en bonne 
partie les stratégies différentes adoptées par les banques commerciales sur le marché secondaire et dans les opérations de réduction négociée de la dette.

Un regard sur le plan Brady révèle ainsi, non seulement la garantie publique à travers les organismes multilatéraux, de la dette convertie après décote négociée, mais aussi la mise en place d'une « division internationale du travail bancaire » entre les grandes banques à projection internationale et celles de moindre dimension qui se replieraient sur leurs marchés nationaux respectifs. Tout ceci dans un scénario de transferts des risques privés vers les créanciers publics. Par ailleurs, les banques d'Europe continentale sont parmi les plus actives à vendre ou à échanger leurs créances sur l'Amérique latine, ce qui s'explique principalement par les législations bancaires nationales favorables à la constitution de réserves pour créances douteuses. Ainsi, les espagnoles ont utilisé le marché secondaire afin d'échanger leurs créances latino-américaines contre des actifs détenus par des banques internationales sur des entreprises espagnoles. Leur volonté de ne plus participer aux "new money » et aux incitations fiscales de l'Etat espagnol offrait des allégements aux banques finançant les entreprises en difficulté, expliquent cette attitude.

Les banques suisses et scandinaves se sont fortement dessaisies de leurs créances sur l'Amérique latine à travers ce marché secondaire, grâce aux approvisionnements très élevés de ces actifs et à la faiblesse de leurs encours. Les désengagements des banques italiennes ont été rendus possibles par le faible niveau absolu de leurs avoirs plus que par le niveau de leurs provisions. Les banques allemandes quant à elles, ont suivi une stratégie sélective : alors que leurs filiales installées au Luxembourg ont été les premières à participer au marché secondaire (surtout dans les titres brésiliens), elles ont été les moins actives sur celui-ci. Il faut voir là une question de politique d'exonération d'impôts permettant de couvrir la dévalorisation de leurs créances latino-américaines. Quoiqu'il en soit, ceci explique qu'à l'heure actuelle les conversions de créances soient devenues le moyen le plus approprié de financement des investissements en Amérique latine (Suarez A., 1991) ${ }^{16}$.

41 Ainsi, par le biais du marché secondaire de la dette notamment, les banques multinationales achèveraient l'intégration productive approfondie car l'implantation des firmes transnationales, principalement dans le secteur automobile (Brésil, Chili, Argentine) et sidérurgique (Venezuela) en est facilitée. De même, parce qu'elles ont tendance à devenir des instruments financiers (moyens de paiement ou de placement), ces créances stimulent l'intégration financière de ces pays, par le développement des marchés locaux de capitaux et de places boursières. Dans cette évolution, le marché secondaire de la dette deviendrait le vecteur d'une nouvelle étape dans les relations Nord-Sud, où l'accumulation dans les pays endettés serait dirigée, plus que dans les années 70, par le capital bancaire international (Suarez A.,1991) ${ }^{17}$.

\section{L'Amérique latine dans le commerce mondial}

Le contexte extérieur est lié à une série de dépendances financières (dette), technologiques, commerciales, culturelles, sanitaires et alimentaires. Reconnaitre la combinaison des facteurs exogènes et endogènes ne signifie nullement que l'analyse de ses impacts ne doive pas être replacée dans le contexte de la polarisation du développement $d u$ capitalisme réellement existant à l'échelle planétaire. Dans cet ensemble, les économies du Sud occupent une place subalterne (Suché Udry, Ch., 1991, Op.cit.) $)^{18}$. 
43 L'interdépendance économique, qui s'est accentuée pendant les 25 dernières années, concerne au premier chef les pays du centre (Europe, Etats-Unis, Japon), tant en termes d'investissements qu'au niveau des échanges entre eux. Il suffit de rappeler que, par comparaison avec les pays sous-développés, les pays développés ne sont pas tenus de recourir aux crédits soumis aux critères du FMI.

Par ailleurs, au début des années 90, le principal facteur de transformation de l'économie mondiale consiste en la consolidation et l'expansion des nouvelles technologies. Une autre tendance d'une importance capitale marque également le contexte international: l'apparition et le renforcement de grands blocs économiques dans le monde développé. La Communauté européenne achève, non sans peine, son processus d'unification, y compris dans le domaine monétaire (1993). Les Etats-Unis et le Canada édifièrent un bloc puissant; certaines économies de la région, particulièrement le Mexique, seront incorporées dans son " orbite gravitationnelle ». Une association de libre-échange entre plusieurs pays du Pacifique est également envisagée.

D'autre part, la détente dans le conflit Est-Ouest développera sans doute le commerce mondial et l'investissement. Toutefois, l'Europe de l'Est et d'autres zones prioritaires pour l'Europe concurrencera l'Amérique latine pour l'obtention de ressources. Il faut également signaler les changements que provoquera dans l'avenir la croissante différenciation économique entre pays en développement. Les nouveaux pays industrialisés du Sud-Est asiatique ont perdu leur position périphérique dans la spécialisation productive mondiale en devenant de gros exportateurs de produits manufacturés. A cet égard, l'Amérique latine et les Caraïbes, en tant que région en développement semi-industrialisée, occupent toujours une position périphérique dans cette spécialisation mondiale, puisque plus de $70 \%$ de leurs exportations (y compris les combustibles) sont constitués de produits primaires (CEPAL, 1990, p. 43) ${ }^{19}$.

\section{Commerce Europe-Amérique latine}

L'échange commercial entre la Communauté européenne et l'Amérique latine confirme cette tendance. En effet, la structure des exportations latino-américaines vers la Communauté européenne est traditionnellement concentrée sur les produits de base et les produits alimentaires $(60 \%)$ et les combustibles $(15 \%)$; les produits manufacturés atteignant à peine $13 \%$ du total proviennent d'un petit nombre de pays (parmi lesquels ressortent le Brésil et le Mexique). Au contraire, les ventes de la Communauté européenne en Amérique latine se concentrent sur les produits industriels. La moitié environ est composée de machines et de matériel de transport, $16 \%$ de produits chimiques et $12 \%$ d'autres produits manufacturés. En termes généraux, l'asymétrie structurelle des échanges commerciaux entre la Communauté et l'Amérique latine s'est maintenue presque intacte durant les 30 dernières années et, à l'heure actuelle, les produits manufacturés à forte valeur ajoutée y occupent une place peu importante (Grandi, J., 1991) $^{20}$.

Ce tableau assez classique des échanges commerciaux entre l'Amérique latine et l'Europe nous rappelle que contrairement à certaines images simplistes, le Nord contrôle la majeure partie des produits agricoles (excédents de céréales de la CE ou des Etats-Unis) et la commercialisation des produits primaires du Sud. Ainsi, la production des deux tiers des matières premières proviennent des pays industrialisés. 
Eric Fottorino, cité par André Udry (1991), auteur d'importants travaux sur les matières premières, a analysé le rapport de la CNUCED (Conférence des Nations unies sur le commerce et le développement), affirme que le Tiers-Monde n'est pas maitre de son destin, mais le grand perdant de l'échange, face à des concurrents puissants qui protègent de mille manières (douanières ou non) les accès aux marchés. L'échange inégal est maintenu, l'appauvrissement par le commerce se poursuit. Si les volumes exportés de café ont progressé de $4 \%$, les revenus ont chuté de près de $22 \%$. Entre 1979 et 1988, l'Amérique latine a accru de $5 \%$ par an en moyenne les volumes exportés vers la Communauté européenne, avec une augmentation de $13 \%$ en 1989, mais la dégradation des termes de l'échange des produits primaires a neutralisé pratiquement tout l'effort exportateur (Grandi, J., op.cit.) ${ }^{21}$.

\section{Dette et désastre écologique}

49 Au cours du dernier quart de siècle, une destruction écologique sans précédent s'est abattue sur tout l'hémisphère. La destruction des forêts tropicales, en particulier, est maintenant bien connue de tous. La dette du Tiers-Monde est -elle la cause de ces phénomènes? Pas exclusivement, ni même peut-être directement, mais la pression qu'elle exerce contribue de manière décisive à les provoquer (Susan George, 1992) ${ }^{22}$.

Sans les flots d'argent facile des années 70 et du début des années 80 , une bonne partie du ravage écologique aurait pu être évitée. Les lourds emprunts des années 70 ont financé d'énormes projets, écologiquement dommageables, tels que barrages géants, centrales nucléaires, usines métallurgiques conçues pour fonctionner au charbon de bois provenant des forêts, complexes industriels et exploitations agricoles gigantesques. Comme nous l'avions dit ci-dessus, les programmes d'ajustement du FMI, conçus pour garantir la restauration d'une balance des paiements positive, encouragent les exportations (afin d'assurer le service de la dette) et induisent de la sorte une surproduction de biens primaires dans le Sud. La première conséquence manifeste en est l'irrésistible pression à la baisse des prix. On sait que les pays en développement, pour assurer le paiement des intérêts de leurs dettes, ont opéré au bénéfice des pays du centre des transferts nets de ressources financières qui atteignent près de 40 milliards de dollars par an (Zantman A., $1990)^{23}$.

51 La deuxième conséquence est d'ordre écologique. Encourager les exportations pourrait être judicieux, s'il n'était donné qu'à quelques pays seulement en même temps. Toutefois des dizaines de pays débiteurs s'évertuent à gagner plus en exportant tout ce qu'ils ont à portée de la main; leurs ressources naturelles en particulier, minerais, cultures tropicales, bois, viande et poisson notamment. Cette approche condamne le continent à une détérioration inévitable des termes de l'échange, à sa marginalisation de l'économie mondiale, à l'exploitation extensive de ses ressources, à la déprédation de ses ressources naturelles et à la dégradation écologique. Autrement dit, le modèle de «croissance tournée vers l'exportation», auquel tiennent tant le Fonds monétaire et la Banque mondiale, est purement extractif, impliquant plus une exploitation de type minier que la gestion et a fortiori de la conservation des ressources. L'orientation vers l'exportation n'encourage pas seulement l'agriculture industrielle à grande échelle, mais favorise aussi l'octroi d'énormes concessions forestières et minières, guidées par le profit à court terme et peu soucieuses de préserver les ressources naturelles. Une telle concentration de richesse et d'avantages dans quelques mains conduit immanquablement au non-respect 
de l'environnement, comme elle mène à la pauvreté et à la marginalisation de la majorité. Et la pauvreté, à son tour, fait planer une grave menace sur l'équilibre écologique. « Nous avons relevé des corrélations positives exceptionnellement fortes entre les niveaux d'endettement élevés et la dégradation de l'environnement, la déforestation en particulier. » (Susan George, 1992, Op.cit.) ${ }^{24}$. C'est la raison pour laquelle déjà en 1987, le premier ministre norvégien Gro Harlen Brundlandt, dans son rapport Notre avenir commun, affirmait que l'écologie est un problème global conduisant les pays riches et pauvres à l'analyse de la corrélation environnement-développement (Ossandon M.,1992) ${ }^{25}$

\section{En guise de conclusion}

L'objet principal de cette contribution a été de considérer l'endettement extérieur latinoaméricain comme intimement lié aux politiques d'ajustements structurels, au commerce extérieur et aux problèmes écologiques que le caractère de l'intégration de ces pays à l'économie mondiale entraînent. Cette approche globale nous semble la plus adéquate dans la mesure où les problèmes du contexte international dépassent l'analyse isolée des mécanismes de la dette dans ses aspects juridiques, économiques et sociaux. Si cette crise apparaît «structurellement terminée » $d u$ point de vue du système financier international, les entraves structurelles restent présentes dans la plupart des pays y compris pour les meilleurs élèves du FMI qui sont parvenus relativement à contrôler les équilibres macro-économiques.

D'une manière générale, l'ensemble des nations du continent restent spécialisés dans l'exportation des biens qui sont précisément les moins dynamiques du commerce international. Paradoxalement, ce sont aussi ces produits qui se heurtent généralement à des barrières spéciales dans les pays développés. Ces mesures protectionnistes sur les marchés des pays développés constituent toujours un facteur d'inhibition vis-à-vis de l'effet potentiellement positif de la croissance dans les transactions commerciales internationales et par rapport aux transformations productives de l'Amérique latine et des Caraïbes.

La restructuration productive internationale restera un cadre de référence pour le continent, bien au-delà de ce que l'on peut obtenir dans les négociations économiques internationales, particulièrement l'Uruguay round du GAIT. En définitive, l'accès aux marchés les plus dynamiques suppose l'incorporation de technologies dont le contrôle est fortement transnationalisé. Il sera difficile de participer dynamiquement à l'expansion de ces marchés, à moins d'un effort régional en recherche et développement, soutenu ou lié à la coopération technique internationale et à l'investissement direct étranger, celui-ci ayant diminué de manière substantielle depuis le début de la crise de la dette extérieure. En dépit de certains succès pour des pays ayant adhéré au plan Brady, qui ont stimulé le retour aux pays de capitaux évadés, le principe suivant lequel il ne suffit pas d'aider, mais qu'il faut surtout investir, reste plus que jamais de mise.

En d'autres termes, si l'Amérique latine ne concentre ses avantages comparatifs que sur ses ressources naturelles et sa main-d'oeuvre à bon marché, elle continuera à accepter des termes d'échanges médiocres. C'est pourquoi le continent devra optimaliser les potentiels endogènes d'efficacité, tant économiques qu'écologiques, en faveur d'une intégration active au marché mondial par des produits et des services de qualité croissante, à haute valeur ajoutée, fournis par l'industrie et les secteurs agricoles 
nationaux. Cela devrait contribuer à créer les conditions pour introduire des technologies à faible consommation d'énergie.

Bref, il s'agit de délier la croissance productive des exportations de la consommation des ressources naturelles, de façon à diminuer la pollution de l'environnement. Parallèlement, resserrer les liens entre les pays d'Amérique latine, dont la dépendance mutuelle augmente de toute manière, permettra de mieux tirer profit d'un marché élargi dans lequel une certaine dose de technologies adaptées devra être envisagée. Le rôle de l'Etat sera donc décisif puisque, par le marché élargi, il gagnera en poids et en stabilité (Lowenthal, P., 1992) ${ }^{26}$. Le marché unique européen constitue à cet égard un véritable défi pour l'Amérique latine, par le haut degré de compétitivité qu'il peut susciter. Pour y accéder, il faudra un considérable effort d'optimisation et de baisse des coûts de production, d'innovation technologique, d'amélioration de la commercialisation (Grandi, J., 1991) ${ }^{27}$.

57 Parallèlement, ces changements nécessiteront sans aucun doute des progrès substantiels dans le renforcement de la démocratie car, historiquement, la mondialisation croissante, entraînant inégalités et dépendance, trouve dans les classes dirigeantes des pays sousdéveloppés des interlocuteurs privilégiés qui acceptent les politiques d'exportations destinées aux pays centraux, qui n'apportent ni croissance ni développement effectif aux vastes couches populaires du continent. C'est pourquoi les gouvernements, mais surtout les sociétés civiles latino-américaines devront élaborer un projet qui envisage une croissance avec équité, une stratégie de la lutte contre la pauvreté et la défense des ressources naturelles dans la recherche de solutions structurelles pour l'Amérique latine. En effet, cinq siècles après la « découverte » de l'Amérique, les paroles de Simon Bolivar, qui souhaitait faire une grande Amérique latine, résonnent encore : «J'ai labouré la mer. Nous ne serons jamais, jamais heureux $»^{28}$.

Espérons que la coopération internationale, mais surtout les peuples latino-américains eux-mêmes, contribueront à renverser cette tendance, car ceux-ci ont aussi le droit d'être heureux.

\section{NOTES}

1. Van Klaveren, A., (1992), «Les relations Europe-Amérique latine: entre l'illusion et le réalisme ", in Cahiers du CERCAL-ULB, n 7, janvier, pp. 11-42.

2. Corm, G., (1992). "L'endettement des pays en voie de développement: origine et mécanismes ", pp. 29-93 in Dette et développement, Editions Publisud, Paris

3. Prebish, R. (1959). International Trade and Payments in an era of coexistence commercial policy in the underdeveloped countries.

4. Ossandon, M., 1991. «Dette, programmes d'ajustement en Amérique latine : point de départ d'une réflexion sur la coopération ", pp. 7-25 in Cahiers du CERCAL-ULB $n^{\circ}$ 6, novembre, "Dette extérieure et environnement en Amérique latine ». Bergel. S (1993). Cahiers du CERCAL, à paraître. 5. Rimez, M. (1991) «La dette extérieure latino-américaine : dix ans de crise ", in Cahiers du CERCAL-ULB $n^{\circ} 2$, mars. 
6. Bien qu'il ne soit pas traité en détail dans cette contribution, les politiques économiques des gouvernements latino-américains orientent l'agriculture et l'industrie vers une course effrénée aux exportations en se fondant sur le principe, élevé au rang de dogme, des avantages comparatifs. Ces principes ont été appliqués à l'extrême par la mise en oeuvre des modèles monétaristes.

7. Singh, A. (1986). « La crise de l'économie mondiale, la stabilisation et l'ajustement structurel : vue d'ensemble », pp. 291-310, in Travail et société. Revue de l'Institut International d'Etudes sociales. Genève, vol. Il, $\mathrm{n}^{\circ} 3$.

8. Ossandon, M., (1985). «Dette latino-américaine : privatisation de flux financiers », in Revue Nouvelle, janvier, pp. 23-40. Ossandon, M (1991), Cahiers CERCAL $\mathrm{n}^{\circ}$ 6, op.cit.

9. Les tenants du NON à Maastricht mettent en avant le caractère uniforme des politiques d'austérité appliquées à l'ensemble de l'Europe et l'apparition inéluctable d'un marché européen à plusieurs vitesses. Voir notamment Cotta Alain (1992). Oui à l'Europe, Non à Maastricht.

10. Marques-Pereira, J. (1992). «Ajustement structurel et réduction de l'intervention sociale de l'Etat », pp. 257-278, in Materiali Vli/2, op.cit.

11. Andre-Udry Ch. (1991). «La mondialisation et le FMI. Une série de trois articles, » in La Brèche $\mathrm{n}^{\circ} 473$, Suisse.

12. Ramonet, I. (1992). «Les rébellions à venir », in Le Monde Diplomatique, Editorial, mars.

13. Van Klaveren, A. (1992). op.cit. Voir aussi sa contribution dans ce numéro.

14. Grandi, J. (1991). «L'impact du marché unique européen sur l'Amérique latine : obstacles et opportunités ", in Cahiers du CERCAL n ${ }^{\circ}$ 7, janvier, pp. 57-72.

15. Suarez, A. (1991). La concurrence interbancaire et la Gestion de la crise de l'endettement latino-américain, in Cahiers du CERCAL-ULB, $\mathrm{n}^{\circ}$ 6, op.cit. novembre, pp. 39-67.

16. Suarez, A. (1991), op.cit.

17. Suarez, A. (1991), op.cit.

18. Andre-Udry, Ch. (1991). op.cit.

19. Rapport CEPAL (1990). Transformacion productiva con equidad, Santiago Chile.

20. Grandi, J. (1991), op.cit.

21. Grandi, J. (1991), op.cit.

22. Susan, G. (1992). Le choc en retour de la dette du Tiers-Monde, L'effet boomerang. Editions la Découverte.

23. Zantman, A. (1990). Le Tiers-Monde dans la crise, la question de la dette et de l'aide extérieure, pp. 347-354, in Le Tiers-Monde,les stratégies de développement à l'épreuve des faits.

24. Susan, G. (1992), op.cit.

25. Ossandon, M. (1992). "Ecologie, dette et commerce : la triple cohérence ", in Cahiers du CERCAL $n^{\circ} 11$, septembre, pp. 47-56. Communication présentée à Rio dans le cadre du CEISAL, juin 1992.

26. Lowenthal, P. (1992). «Ouverture économique et intégration régionale: stratégies complémentaires pour l'Amérique latine ", in Cahiers du CERCAL n ${ }^{\circ} 11$, septembre, pp. 33-46.

27. Grandi, J. (1991). op.cit.

28. Lambert, J., Gandolfi. A. (1987). Le système politique de l'Amérique latine, PUF, Paris. 


\section{RÉSUMÉS}

The autor propose a global approach in analysing the main economic problems that Latin America was confonted with for the past years, prablems such as foreing debt, structural adjustment, obstacles to foreing trade or the destruction of environment, related to the modalities of the region's integration in the international economy. The autor also question himself on the rale that Europe and the european economic actors play in solving or worsening these problems. This way, topics such as the impact of the european neo-protectionismpolicies regarding Latin America exports, the support of governement to adjustment policies within international financial institutions, the particularly active role of certain european banks in the unofficial trading in debts and the conversion of unrepaid loans when repurchasing compagnias or the sharing of costs related to envorenment protection. In response to the often contradictory european policies, the autor argues in favour of a reonforcement of international cooperation, and also of having the Latin-american population taking the responsability of their own destiny.

\section{AUTEUR}

\section{MARCELO OSSANDON}

CERCAL (ULB) 